\title{
Near Zone Hydrodynamics of AdS/CFT Jet Wakes
}

\author{
Jorge Noronha $^{1}$, Miklos Gyulassy ${ }^{1}$, and Giorgio Torrieri ${ }^{2}$ \\ ${ }^{1}$ Department of Physics, Columbia University, 538 West $120^{\text {th }}$ Street, New York, NY \\ 10027, USA \\ ${ }^{2}$ Institut für Theoretische Physik, Johann Wolfgang Goethe Universität, \\ Max von Laue-Str. 1, 60438 Frankfurt am Main, Germany
}

\begin{abstract}
The energy-momentum tensor of a supersonic heavy quark jet moving through a strongly-coupled $\mathcal{N}=4 \mathrm{SYM}$ plasma is analyzed in terms of first-order Navier-Stokes hydrodynamics. We focus on the near zone "head" region close to the heavy quark where deviations from hydrodynamic behavior are expected to be large. For realistic quark velocities, $v=0.99$, we find that the non-hydrodynamical head region is confined at a narrow pancake surrounding the jet with a width $\sim 1 / T$ and transverse radius $\sim 3 / T$. Outside this jet head region, the jet induced stress is well approximated by Navier-Stokes hydrodynamics.

PACS numbers: 25.75.-q, 11.25.Tq, 13.87.-a
\end{abstract}

\section{Introduction}

The suppression of highly energetic particles observed at the Relativistic Heavy Ion Collider (RHIC) [1] suggests that the matter created in the first moments after the two heavy ions have collided is a strongly-coupled deconfined plasma of quarks and gluons [2]. The degree of thermalization of the soft degrees freedom seems to be consistent with the perfect fluid hypothesis where the strongly-coupled quark-gluon plasma (sQGP) has a shear viscosity to entropy density ratio [3] of the order of the string-inspired lower bound $\eta / s=1 /(4 \pi)$ [4] derived using the anti-de Sitter/Conformal Field Theory (AdS/CFT) correspondence [5]. This correspondence provides a framework to calculate nonpertubatively observables associated with strongly-coupled $\mathcal{N}=4$ supersymmetric Yang-Mills (SYM) theories in terms of dual weakly-coupled (super)gravity.

If the RHIC plasma is indeed strongly-coupled, one has to rely on non-perturbative methods, such as AdS/CFT, to describe its properties. At temperatures sufficiently above the critical temperature, the sQGP appears to display some of the inherent properties of $\mathcal{N}=4 \mathrm{SYM}$ plasmas such as conformal invariance. Given the power of AdS/CFT to compute nonequilibrium dynamical quantities, it is natural to investigate whether jet quenching phenomena can be explained in terms of the dual gravitational concepts of AdS/CFT.

In this paper we therefore study the SYM analogous of jet quenching, i.e., the problem of a heavy quark moving through a strongly-coupled $\mathcal{N}=4$ SYM plasma. 
We use the setup proposed in [6] where an infinitely massive heavy quark moving at a constant speed $v$ along the comoving $X_{1}=X-v t$ axis in the SYM plasma corresponds to one of the endpoints of a classical string that lives in a 5-dimensional space defined by an $\mathrm{AdS}_{5}$-Schwarzschild black (brane) hole geometry. In this steady-state solution, the quark has been moving since $t \rightarrow-\infty$ and can be found at the origin at $t=0$. According to the AdS/CFT correspondence, the disturbances in the 5-dimensional metric caused by the string can be used to obtain the full non-perturbative result for the energy-momentum tensor of the system plasma+heavy quark at finite temperature $T$. In Ref. [7], the total energy-momentum tensor of the system was computed analytically to leading order in an expansion in powers of inverse momentum $T / K \ll 1$ and then transformed back to coordinate space. This tensor describes the energy and momentum disturbances induced in the plasma in a spacetime region close to the heavy-quark in the limit where both the t'Hooft coupling constant of the plasma $\lambda$ and the number of colors $N_{c}$ are large. Note that the low-energy excitations found in the linearized analysis of Ref. [8], i.e, the sound and diffusion modes, only appear in the opposite limit where $T / K \gg 1[9]$. In the present work, we compare the analytical result for the stress tensor derived in [7] with a first-order Navier-Stokes ansatz for the energy-momentum tensor in order to determine the distance within which the wake left behind by the heavy quark is thermalized (see also [10] and [11]).

\section{Comparison with first-order Navier Stokes hydrodynamics}

To first order in a gradient expansion, the Navier-Stokes stress tensor is [12]

$$
T_{\mu \nu}^{N S}=(\rho+p) U_{\mu} U_{\nu}+p g_{\mu \nu}+\Pi_{\mu \nu}
$$

where $\rho$ is the local energy density, $p$ is the isotropic pressure, and $U^{\mu}=\left(U^{0}, \vec{U}\right)$ is the flow 4-vector (in our metric $U_{\mu} U^{\mu}=-1$ ). The dissipative contribution to the tensor above is given by the shear tensor (the bulk viscosity of conformal plasmas is identically zero)

$$
\begin{aligned}
\Pi^{\mu \nu} & =-\eta\left(\partial^{\mu} U^{\nu}+\partial^{\nu} U^{\mu}+U^{\mu} U_{\alpha} \partial^{\alpha} U^{\nu}\right. \\
& \left.+U^{\nu} U^{\alpha} \partial_{\alpha} U^{\mu}\right)+\frac{2}{3} \eta \Delta^{\mu \nu}\left(\partial_{\alpha} U^{\alpha}\right),
\end{aligned}
$$

where $\Delta^{\mu \nu}=g^{\mu \nu}+U^{\mu} U^{\nu}$ is the local spatial projector and $\eta=\left(N_{c}-1\right)^{2} \pi T^{3} / 8$. Note that we kept the nonlinear terms in $U^{\mu}$ in the definition of $\Pi_{\mu \nu}$. The flow velocity associated with the full $T_{\mu \nu}^{Y}$ of [7] can be obtained by boosting the tensor to its local rest frame (Landau frame) [10]. The deviation from Navier-Stokes hydrodynamics can then be studied by defining the tensor

$$
Z_{\mu \nu}=T_{\mu \nu}^{Y}-\Pi_{\mu \nu}
$$

and checking its components in the Landau frame. If $\left(T_{\mu \nu}^{Y}\right)_{L}$ (components evaluated in the Landau frame come with the L subscript) can be described by Navier-Stokes, we should obtain

$$
\left(Z_{11}\right)_{L}=\left(Z_{22}\right)_{L}=\left(Z_{33}\right)_{L}=\frac{1}{3}\left(Z_{00}\right)_{L}, \quad\left(Z_{i j}\right)_{L}=0 .
$$



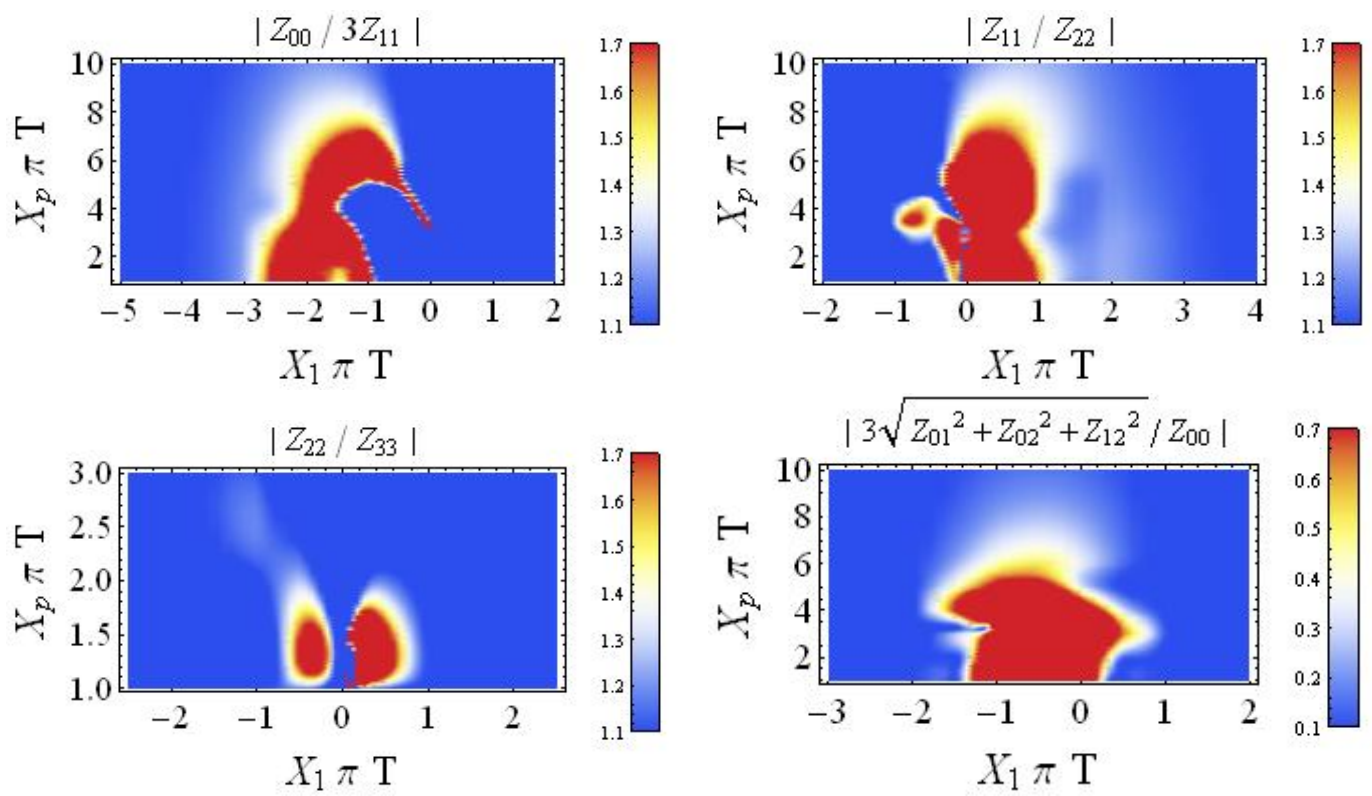

Figure 1. (Color online) $Z_{\mu \nu}$ is defined in Eq. 3. Ratio values outside of the limits were color-coded as the limits.

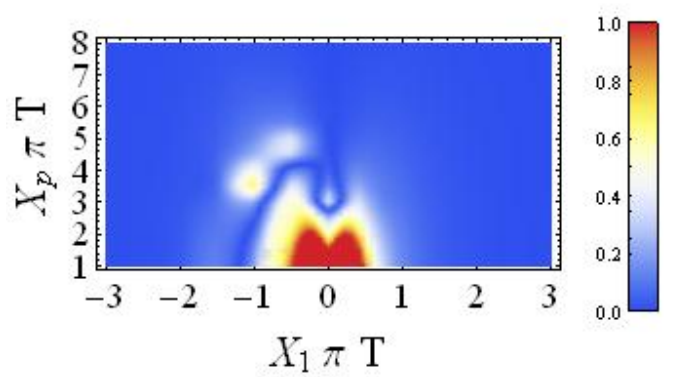

Figure 2. (Color online) Comparison between the full numerical solution for $\vec{U}$ and the leading order result, i.e., $\left|\vec{U}-\vec{S} /\left(4 P_{0}\right)\right|$.

\section{Results}

One can see in Fig. 1 that the magnitude and structure of the discrepancy between Navier-Stokes and $T_{\mu \nu}^{Y}$ remains approximately the same for all the components of $\left(Z_{\mu \nu}\right)_{L}$. We have set $N_{c}=3, \lambda=3 \pi$, and $v=0.99$. Outside the region where $-3<X_{1} \pi T<3$ (longitudinal direction) and $0<X_{p} \pi T<10$ (transverse direction), the system can be described by the first-order Navier-Stokes energy-momentum tensor to an accuracy of roughly $90 \%$. Inside that region, however, the discrepancy quickly increases and eventually diverges. In Fig. 2, one can see that in the region where Navier-Stokes provides a good description of the system the numerically calculated velocity flow $\vec{U}$ matches the leading order result in $1 / N_{c}, \vec{U}=\vec{S} /\left(4 P_{0}\right)$, where $S_{i}=-T_{0 i}^{Y}$ and $P_{0}=\eta \pi T$. Therefore, in this region the system is more accurately described by a linearized version of the first-order Navier-Stokes equations. 


\section{Conclusions}

In this paper we have compared the analytically obtained energy-momentum tensor of an infinitely massive quark moving through a strongly coupled $\mathcal{N}=4 \mathrm{SYM}$ plasma with a non-ideal tensor given by the first-order Navier-Stokes ansatz. We have found that the energy deposited by the quark is thermalized in the region defined by $\left|X_{1}\right| \pi T>3$ and $X_{p} \pi T>1$, which excludes the nonequilibrium jet head. In fact, the system can be described by linearized first-order Navier Stokes hydrodynamics down to distances of roughly $3 /(\pi T)$. Thus, assuming that a strongly coupled $\mathcal{N}=4 \mathrm{SYM}$ plasma can capture the main features of the sQGP, we should expect that the energy deposited by jets induces almost perfect fluid hydrodynamic response of the sQGP outside the head region.

\section{Acknowledgments}

We thank A. Yarom, S. Gubser, and S. Pufu for useful discussions. G.T. thanks the Alexander Von Humboldt foundation for the support provided for this research. J.N. and M.G. acknowledge partial support from DOE under Grant No. DE-FG02-93ER40764 and the ITP and FIAS of the J.W. Goethe University, Frankfurt.

\section{References}

[1] I. Arsene et al. [BRAHMS Collaboration], Nucl. Phys. A 757, 1 (2005); K. Adcox et al. [PHENIX Collaboration], Nucl. Phys. A 757, 184 (2005); B. B. Back et al., Nucl. Phys. A 757, 28 (2005); J. Adams et al. [STAR Collaboration], Nucl. Phys. A 757, 102 (2005).

[2] M. Gyulassy and L. McLerran, Nucl. Phys. A 750, 30 (2005).

[3] U. W. Heinz and P. F. Kolb, Nucl. Phys. A 702, 269 (2002). H. Song and U. W. Heinz, Phys. Lett. B 658, 279 (2008); D. Teaney, J. Lauret and E. V. Shuryak, arXiv:nucl-th/0110037; D. Teaney, Phys. Rev. C 68, 034913 (2003); P. Romatschke and U. Romatschke, Phys. Rev. Lett. 99, 172301 (2007).

[4] G. Policastro, D. T. Son and A. O. Starinets, Phys. Rev. Lett. 87, 081601 (2001); P. Kovtun, D. T. Son and A. O. Starinets, Phys. Rev. Lett. 94, 111601 (2005).

[5] J. M. Maldacena, Adv. Theor. Math. Phys. 2, 231 (1998) [Int. J. Theor. Phys. 38, 1113 (1999)].

[6] C. P. Herzog, A. Karch, P. Kovtun, C. Kozcaz and L. G. Yaffe, JHEP 0607, 013 (2006). S. S. Gubser, Phys. Rev. D 74, 126005 (2006).

[7] A. Yarom, Phys. Rev. D 75, 105023 (2007).

[8] J. Casalderrey-Solana, E. V. Shuryak and D. Teaney, J. Phys. Conf. Ser. 27, 22 (2005) [Nucl. Phys. A 774, 577 (2006)]; J. Casalderrey-Solana, E. V. Shuryak and D. Teaney, arXiv:hep-ph/0602183.

[9] J. J. Friess, S. S. Gubser, G. Michalogiorgakis and S. S. Pufu, Phys. Rev. D 75, 106003 (2007); S. S. Gubser and A. Yarom, Phys. Rev. D 77, 066007 (2008).

[10] J. Noronha, G. Torrieri and M. Gyulassy, arXiv:0712.1053 [hep-ph].

[11] P. M. Chesler and L. G. Yaffe, arXiv:0712.0050 [hep-th].

[12] L. D. Landau, E. M. Lifshitz, Fluid Mechanics (Pergamon Press, New York, 1987), Vol. 6. 